\title{
Chitosan and yeast elicitor in suppressing seed-borne fungi of cucurbitaceous vegetables
}

\author{
Farzana Haque Tumpa, Md. Zahangir Alam, Kawsar Hossen ${ }^{1}$ and Md. Atiqur Rahman Khokon \\ Department of Plant Pathology, Bangladesh Agricultural University, Mymensingh-2202, Bangladesh \\ ${ }^{1}$ Department of Agriculture, Noakhali Science and Technology University, Noakhali-3814, Bangladesh
}

\begin{tabular}{|c|c|}
\hline ARTICLE INFO & Abstract \\
\hline $\begin{array}{l}\text { Article history: } \\
\text { Received: } 26 \text { February } 2018 \\
\text { Accepted: } 07 \text { July } 2018\end{array}$ & \multirow{3}{*}{$\begin{array}{l}\text { Experiments were conducted under laboratory condition to examine the efficacy of Chitosan and Yeast } \\
\text { Elicitor to suppress the growth of seed-borne fungi of cucurbitaceous vegetables. Seeds of bottle gourd, } \\
\text { sweet gourd, snake gourd, wax gourd and cucumber were collected from seed traders of Mymensingh } \\
\text { districts and different seed borne fungi were isolated, purified and identified. Fourteen fungal species } \\
\text { belonging to twelve genera consisting of Aspergillus flavus, Aspergillus niger, Botrytis cinerea, Fusarium } \\
\text { moniliforme, Fusarium oxysporum, Phoma exigua, Rhizopus stolonifer, Macrophomina phaseolina, } \\
\text { Penicillium spp., Curvularia lunata, Chaetomium spp., Colletotrichum spp., Cercospora spp. and } \\
\text { Alternaria alternata were isolated and identified. Four concentrations of Chitosan and Yeast Elicitors } \\
\text { solutions ( } 200,500,1000 \& 2000 \mathrm{ppm}) \text { including one positive control Vitavax- } 200 \mathrm{WP}(0.35 \%) \text { were } \\
\text { evaluated for controlling seed-borne fungi. Among the seed treating agents Chitosan ( } 2000 \mathrm{ppm}) \text { and } \\
\text { Yeast Elicitor ( } 2000 \mathrm{ppm}) \text { showed better performance in suppressing the seed-borne fungi. Chitosan (2000 } \\
\text { ppm) showed superior performance than Yeast Elicitor ( } 2000 \mathrm{ppm}) \text {. Results from the present study } \\
\text { revealed that application of elicitors as seed treatment is a potential alternative of chemical fungicide for } \\
\text { selective vegetables. }\end{array}$} \\
\hline $\begin{array}{l}\text { Keywords: } \\
\text { Chitosan, Yeast elicitor, } \\
\text { Cucurbits, Seed-borne fungi, } \\
\text { Seed priming }\end{array}$ & \\
\hline $\begin{array}{l}\text { Correspondence: } \\
\text { Md. Atiqur Rahman Khokon } \\
\text { (atiq.ppath@bau.edu.bd) }\end{array}$ & \\
\hline
\end{tabular}

\section{Introduction}

Cucurbits are important vegetable crops, not only in Bangladesh, but also in many other countries all over the world. Cucurbits belong to Cucurbitaceae family, which include bottle gourd (Lagenarea siceraria L), sweet gourd (Cucurbita moschata), snake gourd (Trichosanthes cucumeria), wax gourd (Benincasa hispida) and cucumber (Cucumis sativus) etc.

Cucurbits are commonly exposed to attack by many serious soil-borne and seed-borne pathogens. Pathogen free healthy seeds are essential for desired plant populations and a good harvest. Of the $16 \%$ annual crop losses due to plant diseases, at least $10 \%$ loss occurs due to seed-borne diseases (Fakir, 1983). Coincidentally important or devastating crop diseases are seed-borne and caused by fungi. In addition, seed borne fungi are responsible for poor quality seeds in many crops (Neergaard, 1979).

For suppressing seed-borne fungi various elicitors can be used. Yeast Elicitor and Chitosan $(\beta-1,4$ linked Dglucosamine) are two important bio-polymers, can be commercially derived from various crustaceans commonly from the exoskeleton of shrimps and crabs (Boonlertnirun et al., 2008). These two products can modulate various cellular function including reactive oxygen production, ion channel activity through phosphorylation and dephosphorylation of target protein, stomatal movement, upregulation of pathogenesis related genes (Khokon et al. 2010). Both Yeast Elicitor and Chitosan can be used as seed treating agents and foliar application of these components can induce resistance to overcome the seedling diseases as well as final crop production (Mondal et al., 2013). Properties of chitosan for inhibition of pathogenic bacteria and fungi in antimicrobial films and edible coatings are used. Antimicrobial activity of chitosan resulting from positively charged amino groups. This group responds to negatively charged cell membranes of microorganisms. This reaction leads to the leakage of intracellular protein components and other microorganism components (Yarahmadi et al. 2014). Yeast extract (YE) is an important elicitor and is found to be rich in vitamin Bcomplex. It also contains essential components like chitin, N-acetyl-glucosamine oligomers, glucan, glycopeptides and ergosterol (Boller, 1995); these compounds elicit plant defense responses by triggering metabolite synthesis (Putalun et al., 2007; Cai et al., 2012). Yeast Extract has successfully been used in culture and overproduction of important phytocompounds were observed in several studied of plant genera (Prakash and Srivastava, 2008; Zhao et al., 2010; Cai et al., 2012).

Therefore, the objective of the research was to know the potentiality of chitosan and yeast elicitor in suppressing seed-borne fungi isolated from bottle gourd, sweet gourd, snake gourd, wax gourd and cucumber.

\section{Materials and Methods}

The experiments were conducted in the Laboratory of Biosignaling, Bioactive Compounds and Bio 
formulation, Plant Disease Diagnostic Clinic (PDDC), Department of Plant Pathology and Seed Pathology Centre (SPC), Bangladesh Agricultural University, Mymensigh-2202 during the period from October, 2015 to November, 2016.

Seeds of bottle gourd, sweet gourd, snake gourd, wax gourd and cucumber were collected from the farmers of Mymensingh districts. These seeds were stored in ziplock bags in refrigerator for further studies.

Blotter method was followed according to ISTA rules for seed health testing (ISTA, 2006) for detection of seed-borne fungi. After 7 days each individual incubated seed was observed under stereo-binocular microscope at $16 \mathrm{x}$ and $25 \mathrm{x}$ magnifications in order to record the incidence of seed borne fungi. For proper identification of fungi, temporary slides were prepared from the fungal colony and observed under compound microscope and identified with the help of keys suggested by Ellis (1971) and Neergard (1979).

For Chitosan $(0.3 \%$ solution) preparation, $3 \mathrm{~g}$ Chitosan was dissolved in concentrated (98\%) acetic acid diluted by water to a volume of $1000 \mathrm{~mL}$ and $3000 \mathrm{ppm}$ Chitosan stock solution was prepared. From the stock solution 200 ppm, 500 ppm, 1000 ppm \& 2000 ppm chitosan solutions were prepared. For Yeast Elicitor $(0.3 \%$ solution) preparation, Yeast (Saccharomyces cerevisiae) was cultured in YEPDA broth (Yeast extract $1 \%$, Peptone $2 \%$ and Dextrose 2\%). Erlenmeyer flask $(250 \mathrm{~mL})$ having all the ingredients was incubated with shaking on an orbital platform shaker at $30^{\circ} \mathrm{C}$ and 140 rpm for $72 \mathrm{hrs}$ for collecting filtrate from the yeast. After $72 \mathrm{hrs}$ the broth was filtrated and filtrate were collected and mixed with ethanol solution following the key of Ari and Cakir (2009). By this procedure 3000 ppm Yeast Elicitor stock solution was prepared. From the stock solution 200 ppm, 500 ppm, 1000 ppm \& 2000 ppm Yeast Elicitor solution were prepared.

\section{Seed priming with Chitosan and Yeast Elicitor}

Seeds were dipped in respected Chitosan and Yeast Elicitor solution for $2 \mathrm{hrs}$ at room temperature and then seeds were placed in Blotting Paper following ISTA rules for seed testing (ISTA, 2006). As a positive control, seed treatment with Vitavax-200 WP was carried out following the method of Islam et al. (2001).

\section{Statistical analysis}

The data were analyzed following completely randomized design (CRD) with three replications by using the M-STAT C statistical software.

\section{Results}

Effect of Seed Priming with Chitosan and Yeast Elicitor on the Association of Seed-borne Fungi

Total twelve genera of seed borne fungi were observed associated with tested seeds of cucurbits. Fusarium,
Macrophomina, Colletotrichum, Aspergillus, Curvularia, Botrytis, Rhizopus, Phoma, Alternaria, Penicillium, Chaetomium and Cercospora were predominantly associated at various intensity with most of the seed samples (Table 1-5).

In Bottle Gourd, eight fungi viz., Aspergillu flavus, Botrytis cinerea, Aspergillus niger, Fusarium moniliforme, Fusarium oxysporum, Phoma exigua, Rhizopus stolonifer and Macrophomina phaseolina were detected in $\mathrm{T}_{0}$ (Control), while the least seed-borne fungal infections were recorded in $\mathrm{T}_{4}(2000 \mathrm{ppm}$ Chitosan), $T_{7}$ (1000 ppm Yeast Elicitor) and $T_{8}(2000$ ppm Yeast Elicitor) followed by $\mathrm{T}_{3}(1000 \mathrm{ppm}$ Chitosan) and $\mathrm{T}_{6}$ (500 ppm Yeast Elicitor). The prevalence of Aspergillus flavus (13\%) was the most predominant fungus followed by Botrytis cinerea (9.6\%), Aspergillus niger (8.2\%), Fusarium moniliforme $(7.7 \%)$ and Fusarium oxysporum (5.4\%).

In Sweet Gourd, ten fungi viz., Rhizopus stolonifer, Fusarium moniliforme, Aspergillus niger, Phoma exigua, Aspergillus flavus, Macrophomina phaseolina, Botrytis cinerea, Fusarium oxysporum, Penicillium spp. and Curvularia lunata were recorderd in $\mathrm{T}_{0}$ (Control), while the least seed-borne fungal infections were recorded in $T_{4}$ (2000 ppm Chitosan) followed by $T_{6}$ (500 ppm Yeast Elicitor), $\mathrm{T}_{7}$ (1000 ppm Yeast Elicitor) and $\mathrm{T}_{8}$ (2000 ppm Yeast Elicitor). The prevalence of Rhizopus stolonifer (28.1\%) was the most predominant fungus followed by Fusarium moniliforme (12\%), Aspergillus niger (7.4\%), Phoma exigua (3.4\%) and Aspergillus flavus (1.4\%).

In Snake Gourd, eight fungi viz., Fusarium moniliforme, Aspergillus flavus, Fusarium oxysporum, Chaetomium spp., Rhizopus stolonifer, Botrytis cinerea, Aspergillus niger and Macrophomina phaseolina were recorded in $\mathrm{T}_{0}$ (Control), while the least seed-borne fungal infections were recorded in $\mathrm{T}_{4}$ (2000 ppm Chitosan) followed by $\mathrm{T}_{5}$ (200 ppm Yeast Elicitor), $\mathrm{T}_{6}(500 \mathrm{ppm}$ Yeast Elicitor), $\mathrm{T}_{7}$ (1000 ppm Yeast Elicitor) and $\mathrm{T}_{8}$ (2000 ppm Yeast Elicitor). The prevalence of Fusarium moniliforme $(35.6 \%)$ was the most predominant fungus followed by Aspergillus flavus (23.3\%), Fusarium oxysporum (8.2\%), Chaetomium spp. (7.4\%) and Rhizopus stolonifer (5.4\%).

In Wax Gourd, ten fungi viz., Rhizopus stolonifer, Aspergillus niger, Aspergillus flavus, Botrytis cinerea, Macrophomina phaseolina, Phoma exigua, Fusarium moniliforme, Colletotrichum spp., Curvularia lunata and Fusarium oxysporum were detected in $\mathrm{T}_{0}$ (Control), while the least seed-borne fungal infections were recorded in $\mathrm{T}_{4}$ (2000 ppm Chitosan) followed by $\mathrm{T}_{3}$ (1000 ppm Chitosan) and $\mathrm{T}_{8}$ (2000 ppm Yeast Elicitor). The prevalence of Rhizopus stolonifer (15.2\%) was the most predominant fungus followed by Aspergillus niger (11.9\%), Aspergillus flavus (9.3\%) and Botrytis cinerea $(6.9 \%)$. 
In Cucumber, eleven fungi viz., Fusarium moniliforme, Aspergillus flavus, Fusarium oxysporum, Botrytis cinerea, Rhizopus stolonifer, Macrophomina phaseolina, Penicillium spp., Phoma exigua, Colletotrichum spp., Cercospora spp. and Alternaria alternata were observed in $\mathrm{T}_{0}$ (Control), while the least seed-borne fungal infections were recorded in $\mathrm{T}_{8}(2000 \mathrm{ppm}$ Yeast Elicitor), followed by $\mathrm{T}_{4}$ (2000 ppm Chitosan). The prevalence of Fusarium moniliforme (21.1\%) was the most predominant fungus followed by Aspergillus flavus (20.9\%), Fusarium oxysporum $(7.6 \%)$ and Botrytis cinerea $(6.0 \%)$.

Table 1. Effect of Chitosan and Yeast Elicitor on prevalence of seed-borne fungi of bottle gourd

\begin{tabular}{|c|c|c|c|c|c|c|c|c|}
\hline \multirow[t]{2}{*}{ Treatment } & \multicolumn{8}{|c|}{ Prevalence of Seed-borne fungi (\%) } \\
\hline & $\begin{array}{l}\text { Botrytis } \\
\text { cinerea }\end{array}$ & $\begin{array}{c}\text { Fusarium } \\
\text { moniliforme }\end{array}$ & $\begin{array}{c}\text { Macrophomina } \\
\text { phaseolina }\end{array}$ & $\begin{array}{l}\text { Fusarium } \\
\text { oxysporum }\end{array}$ & $\begin{array}{l}\text { Phoma } \\
\text { exigua }\end{array}$ & $\begin{array}{l}\text { Rhizopus } \\
\text { stolonifer }\end{array}$ & $\begin{array}{l}\text { Aspergillus } \\
\text { niger }\end{array}$ & $\begin{array}{l}\text { Aspergillus } \\
\text { flavus }\end{array}$ \\
\hline $\mathrm{T}_{0}$ (Control) & $66 \mathrm{a}$ & $30 \mathrm{a}$ & $\begin{array}{c}4 \mathrm{a} \\
(11.54)\end{array}$ & $24 \mathrm{a}$ & $\begin{array}{c}14 \mathrm{a} \\
(21.97)\end{array}$ & $\begin{array}{c}6 \mathrm{a} \\
(14.18)\end{array}$ & $60 \mathrm{a}$ & $\begin{array}{c}70 \mathrm{a} \\
(56.79)\end{array}$ \\
\hline $\mathrm{T}_{1}(200 \mathrm{ppm} \mathrm{CS})$ & $\begin{array}{c}10 \mathrm{c} \\
(18.43)\end{array}$ & $\begin{array}{c}15 \mathrm{~b} \\
(22.79)\end{array}$ & $\begin{array}{c}0 \mathrm{~b} \\
(0.70)\end{array}$ & $\begin{array}{c}15 \mathrm{~b} \\
(22.70)\end{array}$ & $\begin{array}{c}0 \mathrm{~b} \\
(0.70)\end{array}$ & $\begin{array}{c}4 \mathrm{~b} \\
(11.54)\end{array}$ & $\begin{array}{c}20 \mathrm{~b} \\
(26.57)\end{array}$ & $25 \mathrm{~b}$ \\
\hline $\mathrm{T}_{2}(500 \mathrm{ppm} \mathrm{CS})$ & $\begin{array}{c}0 \mathrm{~d} \\
(0.70)\end{array}$ & $\begin{array}{c}12 \mathrm{c} \\
(20.27)\end{array}$ & $\begin{array}{c}0 \mathrm{~b} \\
(0.70)\end{array}$ & $\begin{array}{c}0 \mathrm{e} \\
(0.70)\end{array}$ & $\begin{array}{c}0 \mathrm{~b} \\
(0.70)\end{array}$ & $\begin{array}{c}3 \mathrm{c} \\
(9.97)\end{array}$ & $\begin{array}{c}2 \mathrm{c} \\
(8.13)\end{array}$ & $\begin{array}{c}10 \mathrm{c} \\
(18.43)\end{array}$ \\
\hline $\mathrm{T}_{3}(1000 \mathrm{ppm} \mathrm{CS})$ & $\begin{array}{c}0 \mathrm{~d} \\
(0.70)\end{array}$ & $\begin{array}{c}10 \mathrm{~d} \\
(18.43)\end{array}$ & $\begin{array}{c}0 \mathrm{~b} \\
(0.70)\end{array}$ & $\begin{array}{c}0 \mathrm{e} \\
(0.70)\end{array}$ & $\begin{array}{c}0 \mathrm{~b} \\
(0.70)\end{array}$ & $\begin{array}{c}0 \mathrm{~d} \\
(0.70)\end{array}$ & $\begin{array}{c}0 \mathrm{~d} \\
(0.70)\end{array}$ & $\begin{array}{c}0 \mathrm{~d} \\
(0.70)\end{array}$ \\
\hline $\mathrm{T}_{4}(2000 \mathrm{ppm} \mathrm{CS})$ & $\begin{array}{c}0 \mathrm{~d} \\
(0.70)\end{array}$ & $\begin{array}{c}0 \mathrm{e} \\
(0.70)\end{array}$ & $\begin{array}{c}0 \mathrm{~b} \\
(0.70)\end{array}$ & $\begin{array}{c}0 \mathrm{e} \\
(0.70)\end{array}$ & $\begin{array}{c}0 \mathrm{~b} \\
(0.70)\end{array}$ & $\begin{array}{c}0 \mathrm{~d} \\
(0.70)\end{array}$ & $\begin{array}{c}0 \mathrm{~d} \\
(0.70)\end{array}$ & $\begin{array}{c}0 \mathrm{~d} \\
(0.70)\end{array}$ \\
\hline $\mathrm{T}_{5}(200 \mathrm{ppm} \mathrm{YES})$ & $\begin{array}{l}20 \mathrm{~b} \\
(26.57)\end{array}$ & $\begin{array}{c}10 \mathrm{~d} \\
(18.43)\end{array}$ & $\begin{array}{c}0 \mathrm{~b} \\
(0.70)\end{array}$ & $\begin{array}{c}10 \mathrm{c} \\
(18.43)\end{array}$ & $\begin{array}{c}0 \mathrm{~b} \\
(0.70)\end{array}$ & $\begin{array}{c}0 \mathrm{~d} \\
(0.70)\end{array}$ & $\begin{array}{c}0 \mathrm{~d} \\
(0.70)\end{array}$ & $25 \mathrm{~b}$ \\
\hline $\mathrm{T}_{6}(500 \mathrm{ppm}$ YES$)$ & $\begin{array}{c}0 \mathrm{~d} \\
(0.70)\end{array}$ & $\begin{array}{c}0 \mathrm{e} \\
(0.70)\end{array}$ & $\begin{array}{c}0 \mathrm{~b} \\
(0.70)\end{array}$ & $\begin{array}{c}5 \mathrm{~d} \\
(12.92)\end{array}$ & $\begin{array}{c}0 \mathrm{~b} \\
(0.70)\end{array}$ & $\begin{array}{c}0 \mathrm{~d} \\
(0.70)\end{array}$ & $\begin{array}{c}0 \mathrm{~d} \\
(0.70)\end{array}$ & $\begin{array}{c}0 \mathrm{~d} \\
(0.70)\end{array}$ \\
\hline $\mathrm{T}_{7}(1000 \mathrm{ppm} \mathrm{YES})$ & $\begin{array}{c}0 \mathrm{~d} \\
(0.70)\end{array}$ & $\begin{array}{c}0 \mathrm{e} \\
(0.70)\end{array}$ & $\begin{array}{c}0 \mathrm{~b} \\
(0.70)\end{array}$ & $\begin{array}{c}0 \mathrm{e} \\
(0.70)\end{array}$ & $\begin{array}{c}0 \mathrm{~b} \\
(0.70)\end{array}$ & $\begin{array}{c}0 \mathrm{~d} \\
(0.70)\end{array}$ & $\begin{array}{c}0 \mathrm{~d} \\
(0.70)\end{array}$ & $\begin{array}{c}0 \mathrm{~d} \\
(0.70)\end{array}$ \\
\hline $\mathrm{T}_{8}(2000 \mathrm{ppm} \mathrm{YES})$ & $\begin{array}{c}0 \mathrm{~d} \\
(0.70)\end{array}$ & $\begin{array}{c}0 \mathrm{e} \\
(0.70)\end{array}$ & $\begin{array}{c}0 \mathrm{~b} \\
(0.70)\end{array}$ & $\begin{array}{c}0 \mathrm{e} \\
(0.70)\end{array}$ & $\begin{array}{c}0 \mathrm{~b} \\
(0.70)\end{array}$ & $\begin{array}{c}0 \mathrm{~d} \\
(0.70)\end{array}$ & $\begin{array}{c}0 \mathrm{~d} \\
(0.70)\end{array}$ & $\begin{array}{c}0 \mathrm{~d} \\
(0.70)\end{array}$ \\
\hline $\mathrm{T}_{9}($ Vitavax-200 WP) & $\begin{array}{c}0 \mathrm{~d} \\
(0.70)\end{array}$ & $\begin{array}{c}0 \mathrm{e} \\
(0.70)\end{array}$ & $\begin{array}{c}0 \mathrm{~b} \\
(0.70)\end{array}$ & $\begin{array}{c}0 \mathrm{e} \\
(0.70)\end{array}$ & $\begin{array}{c}0 \mathrm{~b} \\
(0.70)\end{array}$ & $\begin{array}{c}0 \mathrm{~d} \\
(0.70)\end{array}$ & $\begin{array}{c}0 \mathrm{~d} \\
(0.70)\end{array}$ & $\begin{array}{c}0 \mathrm{~d} \\
(0.70)\end{array}$ \\
\hline $\mathrm{LSD}_{0.05}$ & 1.66 & 1.53 & 0.290 & 0.880 & 0.541 & 0.546 & 0.572 & 0.795 \\
\hline $\mathrm{CV}(\%)$ & 8.41 & 7.95 & 9.73 & 6.29 & 11.24 & 7.92 & 3.22 & 3.61 \\
\hline
\end{tabular}

Values within the same column having a common letter(s) do not differ significantly ( $\mathrm{P} \geq 0.01)$

CS indicates Chitosan Solution, YES indicates Yeast Elicitor Solution

Figures in the parentheses are arcsine transformed values

Table 2. Effect of Chitosn and Yeast Elicitor on prevalence of seed-borne fungi of sweet gourd

\begin{tabular}{|c|c|c|c|c|c|c|c|c|c|c|}
\hline \multirow[t]{2}{*}{ Treatment } & \multicolumn{10}{|c|}{ Prevalence of Seed-borne fungi (\%) } \\
\hline & $\begin{array}{l}\text { Fusarium } \\
\text { moniliforme }\end{array}$ & $\begin{array}{l}\text { Fusarium } \\
\text { oxysporum }\end{array}$ & $\begin{array}{l}\text { Curvularia } \\
\text { lunata }\end{array}$ & $\begin{array}{l}\text { Aspergillus } \\
\text { niger }\end{array}$ & $\begin{array}{l}\text { Aspergillus } \\
\text { flavus }\end{array}$ & $\begin{array}{l}\text { Macrophomina } \\
\text { phaseolina }\end{array}$ & $\begin{array}{l}\text { Rhizopus } \\
\text { stolonifer }\end{array}$ & $\begin{array}{l}\text { Phoma } \\
\text { exigua }\end{array}$ & $\begin{array}{l}\text { Penicillium } \\
\text { spp }\end{array}$ & $\begin{array}{l}\text { Botrytis } \\
\text { cinerea }\end{array}$ \\
\hline $\mathrm{T}_{0}$ (Control) & $30 \mathrm{a}$ & $\begin{array}{c}4 \mathrm{a} \\
(11.54)\end{array}$ & $\begin{array}{c}2 \mathrm{a} \\
(8.13)\end{array}$ & $34 a$ & $\begin{array}{c}6 \mathrm{a} \\
(14.18)\end{array}$ & $\begin{array}{c}12 \mathrm{a} \\
(20.27)\end{array}$ & $56 a$ & $34 a$ & $\begin{array}{c}2 \mathrm{a} \\
(8.13)\end{array}$ & $\begin{array}{c}4 \mathrm{a} \\
(11.54)\end{array}$ \\
\hline $\begin{array}{l}\mathrm{T}_{1}(200 \mathrm{ppm} \\
\mathrm{CS})\end{array}$ & $22 \mathrm{~d}$ & $\begin{array}{l}0 \mathrm{~b} \\
(0.7)\end{array}$ & $\begin{array}{c}0 \mathrm{~b} \\
(0.7)\end{array}$ & $25 \mathrm{~b}$ & $\begin{array}{c}4 \mathrm{~b} \\
(11.54)\end{array}$ & $\begin{array}{l}0 \mathrm{~b} \\
(0.7)\end{array}$ & $40 \mathrm{~b}$ & $\begin{array}{c}0 \mathrm{~b} \\
(0.7)\end{array}$ & $\begin{array}{c}1 \mathrm{~b} \\
(5.74)\end{array}$ & $\begin{array}{c}0 \mathrm{c} \\
(0.7)\end{array}$ \\
\hline $\begin{array}{l}\mathrm{T}_{2}(500 \mathrm{ppm} \\
\mathrm{CS})\end{array}$ & $21 \mathrm{e}$ & $\begin{array}{l}0 \mathrm{~b} \\
(0.7)\end{array}$ & $\begin{array}{l}0 \mathrm{~b} \\
(0.7)\end{array}$ & $\begin{array}{c}10 \mathrm{c} \\
(18.43)\end{array}$ & $\begin{array}{c}3 c \\
(9.97)\end{array}$ & $\begin{array}{l}0 \mathrm{~b} \\
(0.7)\end{array}$ & $35 \mathrm{c}$ & $\begin{array}{l}0 \mathrm{~b} \\
(0.7)\end{array}$ & $\begin{array}{c}0 \mathrm{c} \\
(0.7)\end{array}$ & $\begin{array}{l}0 \mathrm{c} \\
(0.7)\end{array}$ \\
\hline $\begin{array}{l}\mathrm{T}_{3}(1000 \mathrm{ppm} \\
\mathrm{CS})\end{array}$ & $\begin{array}{c}20 \mathrm{~b} \\
(26.56)\end{array}$ & $\begin{array}{l}0 \mathrm{~b} \\
(0.7)\end{array}$ & $\begin{array}{l}0 \mathrm{~b} \\
(0.7)\end{array}$ & $\begin{array}{c}5 \mathrm{~d} \\
(12.92)\end{array}$ & $\begin{array}{c}0 \mathrm{e} \\
(0.7)\end{array}$ & $\begin{array}{l}0 \mathrm{~b} \\
(0.7)\end{array}$ & $30 \mathrm{~d}$ & $\begin{array}{l}0 \mathrm{~b} \\
(0.7)\end{array}$ & $\begin{array}{c}0 \mathrm{c} \\
(0.7)\end{array}$ & $\begin{array}{l}0 \mathrm{c} \\
(0.7)\end{array}$ \\
\hline $\begin{array}{l}\mathrm{T}_{4}(2000 \mathrm{ppm} \\
\mathrm{CS})\end{array}$ & $\begin{array}{l}2 \mathrm{~g} \\
(8.13)\end{array}$ & $\begin{array}{l}0 \mathrm{~b} \\
(0.7)\end{array}$ & $\begin{array}{l}0 \mathrm{~b} \\
(0.7)\end{array}$ & $\begin{array}{c}0 \mathrm{e} \\
(0.7)\end{array}$ & $\begin{array}{l}0 \mathrm{e} \\
(0.7)\end{array}$ & $\begin{array}{l}0 \mathrm{~b} \\
(0.7)\end{array}$ & $\begin{array}{c}5 \mathrm{~g} \\
(12.92)\end{array}$ & $\begin{array}{l}0 \mathrm{~b} \\
(0.7)\end{array}$ & $\begin{array}{l}0 \mathrm{c} \\
(0.7)\end{array}$ & $\begin{array}{c}0 \mathrm{c} \\
(0.7)\end{array}$ \\
\hline $\begin{array}{l}\mathrm{T}_{5}(200 \mathrm{ppm} \\
\text { YES) }\end{array}$ & $\begin{array}{c}20 \mathrm{~b} \\
(26.56)\end{array}$ & $\begin{array}{l}0 \mathrm{~b} \\
(0.7)\end{array}$ & $\begin{array}{l}0 \mathrm{~b} \\
(0.7)\end{array}$ & $\begin{array}{c}0 \mathrm{e} \\
(0.7)\end{array}$ & $\begin{array}{c}3 \mathrm{c} \\
(9.97)\end{array}$ & $\begin{array}{l}0 \mathrm{~b} \\
(0.7)\end{array}$ & $30 \mathrm{~d}$ & $\begin{array}{l}0 \mathrm{~b} \\
(0.7)\end{array}$ & $\begin{array}{c}0 \mathrm{c} \\
(0.7)\end{array}$ & $\begin{array}{c}2 \mathrm{~b} \\
(8.13)\end{array}$ \\
\hline $\begin{array}{l}\mathrm{T}_{6}(500 \mathrm{ppm} \\
\text { YES) }\end{array}$ & $\begin{array}{c}15 \mathrm{c} \\
(22.79)\end{array}$ & $\begin{array}{l}0 \mathrm{~b} \\
(0.7)\end{array}$ & $\begin{array}{l}0 \mathrm{~b} \\
(0.7)\end{array}$ & $\begin{array}{l}0 \mathrm{e} \\
(0.7)\end{array}$ & $\begin{array}{l}0 \mathrm{e} \\
(0.7)\end{array}$ & $\begin{array}{l}0 \mathrm{~b} \\
(0.7)\end{array}$ & $25 \mathrm{e}$ & $\begin{array}{l}0 \mathrm{~b} \\
(0.7)\end{array}$ & $\begin{array}{c}0 \mathrm{c} \\
(0.7)\end{array}$ & $\begin{array}{c}0 \mathrm{c} \\
(0.7)\end{array}$ \\
\hline $\mathrm{T}_{7}(1000 \mathrm{ppm}$ & $10 \mathrm{f}$ & $0 \mathrm{~b}$ & $0 \mathrm{~b}$ & $0 \mathrm{e}$ & $0 \mathrm{e}$ & $0 \mathrm{~b}$ & $20 \mathrm{e}$ & $0 \mathrm{~b}$ & $0 \mathrm{c}$ & $0 \mathrm{c}$ \\
\hline YES) & (18.43) & $(0.7)$ & $(0.7)$ & $(0.7)$ & $(0.7)$ & $(0.7)$ & $(26.56)$ & $(0.7)$ & $(0.7)$ & $(0.7)$ \\
\hline $\mathrm{T}_{8}(2000 \mathrm{ppm}$ & $2 \mathrm{~g}$ & $0 \mathrm{~b}$ & $0 \mathrm{~b}$ & $0 \mathrm{e}$ & $0 \mathrm{e}$ & $0 \mathrm{~b}$ & $15 \mathrm{f}$ & $0 \mathrm{~b}$ & $0 \mathrm{c}$ & $0 \mathrm{c}$ \\
\hline YES) & $(8.13)$ & $(0.7)$ & $(0.7)$ & $(0.7)$ & $(0.7)$ & $(0.7)$ & (22.79) & $(0.7)$ & $(0.7)$ & $(0.7)$ \\
\hline $\begin{array}{l}\mathrm{T}_{9} \text { (Vitavax- } \\
200 \mathrm{WP})\end{array}$ & $\begin{array}{c}0 \mathrm{~h} \\
(0.7)\end{array}$ & $\begin{array}{l}0 \mathrm{~b} \\
(0.7)\end{array}$ & $\begin{array}{c}0 \mathrm{~b} \\
(0.7)\end{array}$ & $\begin{array}{c}0 \mathrm{e} \\
(0.7)\end{array}$ & $\begin{array}{c}1 \mathrm{~d} \\
(5.74)\end{array}$ & $\begin{array}{c}0 \mathrm{~b} \\
(0.7)\end{array}$ & $25 \mathrm{e}$ & $\begin{array}{l}0 \mathrm{~b} \\
(0.7)\end{array}$ & $\begin{array}{c}0 \mathrm{c} \\
(0.7)\end{array}$ & $\begin{array}{l}0 \mathrm{c} \\
(0.7)\end{array}$ \\
\hline $\mathrm{LSD}_{0.05}$ & 0.592 & 0.541 & 0.076 & 1.10 & 0.832 & 1.07 & 1.86 & 1.08 & 0.544 & 0.295 \\
\hline CV $(\%)$ & 1.89 & 17.81 & 3.05 & 6.84 & 8.91 & 23.80 & 3.61 & 15.71 & 16.38 & 6.87 \\
\hline
\end{tabular}

Values within the same column having a common letter(s) do not differ significantly ( $\mathrm{P} \geq 0.01)$

CS indicates Chitosan Solution, YES indicates Yeast Elicitor Solution

Figures in the parentheses are arcsine transformed values 
Table 3. Effect of Chitosn and Yeast Elicitor on prevalence of seed-borne fungi of snake gourd seeds

\begin{tabular}{|c|c|c|c|c|c|c|c|c|}
\hline \multirow[t]{2}{*}{ Treatment } & \multicolumn{8}{|c|}{ Prevalence of Seed-borne fungi $(\%)$} \\
\hline & $\begin{array}{l}\text { Aspergillus } \\
\text { flavus }\end{array}$ & $\begin{array}{l}\text { Fusarium } \\
\text { oxysporum }\end{array}$ & $\begin{array}{c}\text { Fusarium } \\
\text { moniliforme }\end{array}$ & $\begin{array}{l}\text { Aspergillus } \\
\text { niger }\end{array}$ & $\begin{array}{l}\text { Rhizopus } \\
\text { stolonifer }\end{array}$ & $\begin{array}{c}\text { Macrophomina } \\
\text { phaseolina }\end{array}$ & $\begin{array}{l}\text { Botrytis } \\
\text { cinerea }\end{array}$ & $\begin{array}{c}\text { Chaetomium } \\
\text { spp }\end{array}$ \\
\hline $\mathrm{T}_{0}$ (Control) & $68 \mathrm{a}$ & $42 \mathrm{a}$ & $\begin{array}{c}76 \mathrm{a} \\
(60.67)\end{array}$ & $\begin{array}{c}18 \mathrm{a} \\
(25.10)\end{array}$ & $\begin{array}{c}8 \mathrm{a} \\
(16.43)\end{array}$ & $\begin{array}{c}2 \mathrm{a} \\
(8.13)\end{array}$ & $42 \mathrm{a}$ & $\begin{array}{c}74 a \\
(59.34)\end{array}$ \\
\hline $\mathrm{T}_{1}(200 \mathrm{ppm} \mathrm{CS})$ & $45 b$ & $\begin{array}{c}10 \mathrm{c} \\
(18.43)\end{array}$ & $50 \mathrm{~b}$ & $\begin{array}{c}15 b \\
(22.79)\end{array}$ & $\begin{array}{c}8 \mathrm{a} \\
(16.43)\end{array}$ & $\begin{array}{c}0 \mathrm{~b} \\
(0.70)\end{array}$ & $\begin{array}{c}0 \mathrm{~b} \\
(0.70)\end{array}$ & $\begin{array}{c}0 \mathrm{~b} \\
(0.70)\end{array}$ \\
\hline $\mathrm{T}_{2}(500 \mathrm{ppm} \mathrm{CS})$ & $40 \mathrm{c}$ & $\begin{array}{c}0 \mathrm{~d} \\
(0.70)\end{array}$ & $45 \mathrm{c}$ & $\begin{array}{c}5 \mathrm{c} \\
(12.92)\end{array}$ & $\begin{array}{c}7 \mathrm{a} \\
(15.34)\end{array}$ & $\begin{array}{c}0 \mathrm{~b} \\
(0.70)\end{array}$ & $\begin{array}{c}0 \mathrm{~b} \\
(0.70)\end{array}$ & $\begin{array}{c}0 \mathrm{~b} \\
(0.70)\end{array}$ \\
\hline $\mathrm{T}_{3}(1000 \mathrm{ppm} \mathrm{CS})$ & $35 \mathrm{~d}$ & $\begin{array}{c}0 \mathrm{~d} \\
(0.70)\end{array}$ & $\begin{array}{c}15 f \\
(22.79)\end{array}$ & $\begin{array}{c}0 \mathrm{~d} \\
(0.70)\end{array}$ & $\begin{array}{c}3 \mathrm{c} \\
(9.97)\end{array}$ & $\begin{array}{c}0 \mathrm{~b} \\
(0.70)\end{array}$ & $\begin{array}{c}0 \mathrm{~b} \\
(0.70)\end{array}$ & $\begin{array}{c}0 \mathrm{~b} \\
(0.70)\end{array}$ \\
\hline $\mathrm{T}_{4}(2000 \mathrm{ppm} \mathrm{CS})$ & $\begin{array}{c}0 \mathrm{e} \\
(0.70)\end{array}$ & $\begin{array}{c}0 \mathrm{~d} \\
(0.70)\end{array}$ & $\begin{array}{c}0 \mathrm{~g} \\
(0.70)\end{array}$ & $\begin{array}{c}0 \mathrm{~d} \\
(0.70)\end{array}$ & $\begin{array}{c}0 \mathrm{~d} \\
(0.70)\end{array}$ & $\begin{array}{c}0 \mathrm{~b} \\
(0.70)\end{array}$ & $\begin{array}{c}0 \mathrm{~b} \\
(0.70)\end{array}$ & $\begin{array}{c}0 \mathrm{~b} \\
(0.70)\end{array}$ \\
\hline $\mathrm{T}_{5}(200 \mathrm{ppm}$ YES$)$ & $\begin{array}{c}0 \mathrm{e} \\
(0.70)\end{array}$ & $\begin{array}{c}0 \mathrm{~d} \\
(0.70)\end{array}$ & $50 \mathrm{~b}$ & $\begin{array}{c}0 \mathrm{~d} \\
(0.70)\end{array}$ & $\begin{array}{c}8 \mathrm{a} \\
(16.43)\end{array}$ & $\begin{array}{c}0 \mathrm{~b} \\
(0.70)\end{array}$ & $\begin{array}{c}0 \mathrm{~b} \\
(0.70)\end{array}$ & $\begin{array}{c}0 \mathrm{~b} \\
(0.70)\end{array}$ \\
\hline $\mathrm{T}_{6}(500 \mathrm{ppm} \mathrm{YES})$ & $\begin{array}{c}0 \mathrm{e} \\
(0.70)\end{array}$ & $\begin{array}{c}0 \mathrm{~d} \\
(0.70)\end{array}$ & $45 \mathrm{c}$ & $\begin{array}{c}0 \mathrm{~d} \\
(0.70)\end{array}$ & $\begin{array}{c}7 \mathrm{a} \\
(15.34)\end{array}$ & $\begin{array}{c}0 \mathrm{~b} \\
(0.70)\end{array}$ & $\begin{array}{c}0 \mathrm{~b} \\
(0.70)\end{array}$ & $\begin{array}{c}0 \mathrm{~b} \\
(0.70)\end{array}$ \\
\hline $\mathrm{T}_{7}(1000 \mathrm{ppm} \mathrm{YES})$ & $\begin{array}{c}0 \mathrm{e} \\
(0.70)\end{array}$ & $\begin{array}{c}0 \mathrm{~d} \\
(0.70)\end{array}$ & $40 \mathrm{~d}$ & $\begin{array}{c}0 \mathrm{~d} \\
(0.70)\end{array}$ & $\begin{array}{c}5 \mathrm{~b} \\
(12.92)\end{array}$ & $\begin{array}{c}0 \mathrm{~b} \\
(0.70)\end{array}$ & $\begin{array}{c}0 \mathrm{~b} \\
(0.70)\end{array}$ & $\begin{array}{c}0 \mathrm{~b} \\
(0.70)\end{array}$ \\
\hline $\mathrm{T}_{8}(2000 \mathrm{ppm} \mathrm{YES})$ & $\begin{array}{c}0 \mathrm{e} \\
(0.70)\end{array}$ & $\begin{array}{c}0 \mathrm{~d} \\
(0.70)\end{array}$ & $35 \mathrm{e}$ & $\begin{array}{c}0 \mathrm{~d} \\
(0.70)\end{array}$ & $\begin{array}{c}4 \mathrm{~b} \\
(11.54)\end{array}$ & $\begin{array}{c}0 \mathrm{~b} \\
(0.70)\end{array}$ & $\begin{array}{c}0 \mathrm{~b} \\
(0.70)\end{array}$ & $\begin{array}{c}0 \mathrm{~b} \\
(0.70)\end{array}$ \\
\hline $\mathrm{T}_{9}($ Vitavax-200 WP) & $45 b$ & $30 \mathrm{~b}$ & $\begin{array}{c}0 \mathrm{~g} \\
(0.70)\end{array}$ & $\begin{array}{c}0 \mathrm{~d} \\
(0.70)\end{array}$ & $\begin{array}{c}4 \mathrm{~b} \\
(11.54)\end{array}$ & $\begin{array}{c}0 \mathrm{~b} \\
(0.70)\end{array}$ & $\begin{array}{c}0 \mathrm{~b} \\
(0.70)\end{array}$ & $\begin{array}{c}0 \mathrm{~b} \\
(0.70)\end{array}$ \\
\hline $\mathrm{LSD}_{0.05}$ & 1.31 & 1.10 & 2.01 & 1.25 & 1.44 & 0.093 & 1.18 & 1.23 \\
\hline CV $(\%)$ & 3.28 & 6.79 & 3.38 & 11.19 & 6.70 & 3.59 & 14.42 & 11.08 \\
\hline
\end{tabular}

Values within the same column having a common letter(s) do not differ significantly ( $\mathrm{P} \geq 0.01)$

CS indicates Chitosan Solution, YES indicates Yeast Elicitor Solution

Figures in the parentheses are arcsine transformed values

Table 4. Effect of Chitosn and Yeast Elicitor on prevalence of seed-borne fungi of wax gourd

\begin{tabular}{|c|c|c|c|c|c|c|c|c|c|c|}
\hline \multirow[t]{2}{*}{ Treatment } & \multicolumn{10}{|c|}{ Percent Prevalence of Seed-borne fungi (\%) } \\
\hline & $\begin{array}{l}\text { Fusarium } \\
\text { oxysporum }\end{array}$ & $\begin{array}{l}\text { Macrophominaph } \\
\text { aseolina }\end{array}$ & $\begin{array}{l}\text { Aspergillus } \\
\text { niger }\end{array}$ & $\begin{array}{l}\text { Rhizopus } \\
\text { stolonifer }\end{array}$ & $\begin{array}{l}\text { Fusarium } \\
\text { moniliforme }\end{array}$ & $\begin{array}{l}\text { Curvularia } \\
\text { lunata }\end{array}$ & $\begin{array}{l}\text { Phoma } \\
\text { exigua }\end{array}$ & $\begin{array}{l}\text { Colletotrichum } \\
\text { spp }\end{array}$ & $\begin{array}{l}\text { Aspergillus } \\
\text { flavus }\end{array}$ & $\begin{array}{l}\text { Botrytis } \\
\text { cinerea }\end{array}$ \\
\hline $\mathrm{T}_{0}$ (Control) & $\begin{array}{c}12 \mathrm{a} \\
(20.27)\end{array}$ & $40 \mathrm{a}$ & $54 \mathrm{a}$ & $52 \mathrm{a}$ & $\begin{array}{c}8 \mathrm{a} \\
(16.43)\end{array}$ & $\begin{array}{c}18 \mathrm{a} \\
(25.10)\end{array}$ & $38 \mathrm{a}$ & $24 a$ & $22 a$ & $24 \mathrm{~b}$ \\
\hline $\begin{array}{l}\mathrm{T}_{1}(200 \mathrm{ppm} \\
\mathrm{CS})\end{array}$ & $\begin{array}{c}0 \mathrm{~b} \\
(0.70)\end{array}$ & $\begin{array}{c}0 \mathrm{~b} \\
(0.70)\end{array}$ & $40 \mathrm{~b}$ & $\begin{array}{c}15 d \\
(22.79)\end{array}$ & $\begin{array}{c}2 \mathrm{e} \\
(8.13)\end{array}$ & $\begin{array}{c}0 \mathrm{~b} \\
(0.70)\end{array}$ & $\begin{array}{c}0 \mathrm{~b} \\
(0.70)\end{array}$ & $\begin{array}{c}0 \mathrm{~b} \\
(0.70)\end{array}$ & $\begin{array}{c}10 \mathrm{c} \\
(18.43)\end{array}$ & $\begin{array}{c}0 \mathrm{e} \\
(0.70)\end{array}$ \\
\hline $\begin{array}{l}\mathrm{T}_{2}(500 \mathrm{ppm} \\
\mathrm{CS})\end{array}$ & $\begin{array}{c}0 \mathrm{~b} \\
(0.70)\end{array}$ & $\begin{array}{c}0 \mathrm{~b} \\
(0.70)\end{array}$ & $\begin{array}{c}20 \mathrm{c} \\
(26.56)\end{array}$ & $\begin{array}{c}10 \mathrm{e} \\
(18.43)\end{array}$ & $\begin{array}{c}1 \mathrm{f} \\
(5.74)\end{array}$ & $\begin{array}{c}0 \mathrm{~b} \\
(0.70)\end{array}$ & $\begin{array}{c}0 \mathrm{~b} \\
(0.70)\end{array}$ & $\begin{array}{c}0 \mathrm{~b} \\
(0.70)\end{array}$ & $\begin{array}{c}8 d \\
(16.43)\end{array}$ & $\begin{array}{c}0 \mathrm{e} \\
(0.70)\end{array}$ \\
\hline $\begin{array}{l}\mathrm{T}_{3}(1000 \mathrm{ppm} \\
\text { CS) }\end{array}$ & $\begin{array}{c}0 \mathrm{~b} \\
(0.70)\end{array}$ & $\begin{array}{c}0 \mathrm{~b} \\
(0.70)\end{array}$ & $\begin{array}{c}5 \mathrm{~d} \\
(12.92)\end{array}$ & $\begin{array}{c}\text { Of } \\
(0.70)\end{array}$ & $\begin{array}{c}0 \mathrm{~g} \\
(0.70)\end{array}$ & $\begin{array}{c}0 \mathrm{~b} \\
(0.70)\end{array}$ & $\begin{array}{c}0 \mathrm{~b} \\
(0.70)\end{array}$ & $\begin{array}{c}0 \mathrm{~b} \\
(0.70)\end{array}$ & $\begin{array}{c}6 \mathrm{e} \\
(14.18)\end{array}$ & $\begin{array}{c}0 \mathrm{e} \\
(0.70)\end{array}$ \\
\hline $\begin{array}{l}\mathrm{T}_{4}(2000 \mathrm{ppm} \\
\mathrm{CS})\end{array}$ & $\begin{array}{c}0 \mathrm{~b} \\
(0.70)\end{array}$ & $\begin{array}{c}0 \mathrm{~b} \\
(0.70)\end{array}$ & $\begin{array}{c}0 \mathrm{e} \\
(0.70)\end{array}$ & $\begin{array}{c}\text { Of } \\
(0.70)\end{array}$ & $\begin{array}{c}0 \mathrm{~g} \\
(0.70)\end{array}$ & $\begin{array}{c}0 \mathrm{~b} \\
(0.70)\end{array}$ & $\begin{array}{c}0 \mathrm{~b} \\
(0.70)\end{array}$ & $\begin{array}{c}0 \mathrm{~b} \\
(0.70)\end{array}$ & $\begin{array}{c}5 f \\
(12.92)\end{array}$ & $\begin{array}{c}0 \mathrm{e} \\
(0.70)\end{array}$ \\
\hline $\begin{array}{l}\mathrm{T}_{5}(200 \mathrm{ppm} \\
\text { YES) }\end{array}$ & $\begin{array}{c}0 \mathrm{~b} \\
(0.70)\end{array}$ & $\begin{array}{c}0 \mathrm{~b} \\
(0.70)\end{array}$ & $\begin{array}{c}0 \mathrm{e} \\
(0.70)\end{array}$ & $30 \mathrm{~b}$ & $\begin{array}{c}8 \mathrm{a} \\
(16.43)\end{array}$ & $\begin{array}{c}0 \mathrm{~b} \\
(0.70)\end{array}$ & $\begin{array}{c}0 \mathrm{~b} \\
(0.70)\end{array}$ & $\begin{array}{c}0 \mathrm{~b} \\
(0.70)\end{array}$ & $\begin{array}{c}15 \mathrm{a} \\
(22.79)\end{array}$ & $\begin{array}{c}20 \mathrm{a} \\
(26.56)\end{array}$ \\
\hline $\begin{array}{l}\mathrm{T}_{6}(500 \mathrm{ppm} \\
\text { YES) }\end{array}$ & $\begin{array}{c}0 \mathrm{~b} \\
(0.70)\end{array}$ & $\begin{array}{c}0 \mathrm{~b} \\
(0.70)\end{array}$ & $\begin{array}{c}0 \mathrm{e} \\
(0.70)\end{array}$ & $25 \mathrm{c}$ & $\begin{array}{c}7 \mathrm{~b} \\
(15.34)\end{array}$ & $\begin{array}{c}0 \mathrm{~b} \\
(0.70)\end{array}$ & $\begin{array}{c}0 \mathrm{~b} \\
(0.70)\end{array}$ & $\begin{array}{c}0 \mathrm{~b} \\
(0.70)\end{array}$ & $\begin{array}{c}12 b \\
020.27\end{array}$ & $\begin{array}{c}15 \mathrm{c} \\
(22.79)\end{array}$ \\
\hline $\begin{array}{l}\mathrm{T}_{7}(1000 \mathrm{ppm} \\
\text { YES) }\end{array}$ & $\begin{array}{c}0 \mathrm{~b} \\
(0.70)\end{array}$ & $\begin{array}{c}0 \mathrm{~b} \\
(0.70)\end{array}$ & $\begin{array}{c}0 \mathrm{e} \\
(0.70)\end{array}$ & $\begin{array}{c}20 \mathrm{c} \\
(26.56)\end{array}$ & $\begin{array}{c}6 c \\
(14.18)\end{array}$ & $\begin{array}{c}0 \mathrm{~b} \\
(0.70)\end{array}$ & $\begin{array}{c}0 \mathrm{~b} \\
(0.70)\end{array}$ & $\begin{array}{c}0 \mathrm{~b} \\
(0.70)\end{array}$ & $\begin{array}{c}10 \mathrm{c} \\
(18.43)\end{array}$ & $\begin{array}{c}10 \mathrm{~d} \\
(18.43)\end{array}$ \\
\hline $\begin{array}{l}\mathrm{T}_{8}(2000 \mathrm{ppm} \\
\text { YES) }\end{array}$ & $\begin{array}{c}0 \mathrm{~b} \\
(0.70)\end{array}$ & $\begin{array}{c}0 \mathrm{~b} \\
(0.70)\end{array}$ & $\begin{array}{c}0 \mathrm{e} \\
(0.70)\end{array}$ & $\begin{array}{c}\text { Of } \\
(0.70)\end{array}$ & $\begin{array}{c}3 \mathrm{~d} \\
(9.97)\end{array}$ & $\begin{array}{c}0 \mathrm{~b} \\
(0.70)\end{array}$ & $\begin{array}{c}0 \mathrm{~b} \\
(0.70)\end{array}$ & $\begin{array}{c}0 \mathrm{~b} \\
(0.70)\end{array}$ & $5 \mathrm{~g}$ & $\begin{array}{c}0 \mathrm{e} \\
(0.70)\end{array}$ \\
\hline $\begin{array}{l}\mathrm{T}_{9} \text { (Vitavax- } \\
200 \mathrm{WP} \text { ) }\end{array}$ & $\begin{array}{c}0 \mathrm{~b} \\
(0.70)\end{array}$ & $\begin{array}{c}0 \mathrm{~b} \\
(0.70)\end{array}$ & $\begin{array}{c}0 \mathrm{e} \\
(0.70)\end{array}$ & $\begin{array}{c}\text { Of } \\
(0.70)\end{array}$ & $\begin{array}{c}0 \mathrm{~g} \\
(0.70)\end{array}$ & $\begin{array}{c}0 \mathrm{~b} \\
(0.70)\end{array}$ & $\begin{array}{c}0 \mathrm{~b} \\
(0.70)\end{array}$ & $\begin{array}{c}0 b \\
(0.70)\end{array}$ & $\begin{array}{c}0 \mathrm{~h} \\
(0.70)\end{array}$ & $\begin{array}{c}0 \mathrm{e} \\
(0.70)\end{array}$ \\
\hline $\mathrm{LSD}_{0.05}$ & 0.685 & 1.08 & 1.24 & 1.63 & 0.807 & 0.594 & 1.35 & 1.29 & 1.12 & 1.14 \\
\hline CV (\%) & 15.16 & 13.73 & 5.29 & 5.40 & 5.37 & 11.12 & 17.86 & 25.13 & 4.36 & 6.99 \\
\hline
\end{tabular}

Values within the same column having a common letter(s) do not differ significantly ( $\mathrm{P} \geq 0.01)$

CS indicates Chitosan Solution, YES indicates Yeast Elicitor Solution

Figures in the parentheses are arcsine transformed values 
Table 5. Effect of Chitosn and Yeast Elicitor on prevalence of seed-borne fungi of cucumber

\begin{tabular}{|c|c|c|c|c|c|c|c|c|c|c|c|}
\hline \multirow[t]{2}{*}{ Treatment } & \multicolumn{11}{|c|}{ Percent Prevalence of Seed-borne fungi $(\%)$} \\
\hline & $\begin{array}{l}\text { Botrytis } \\
\text { cinerea }\end{array}$ & $\begin{array}{c}\text { Fusarium } \\
\text { moniliforme }\end{array}$ & $\begin{array}{l}\text { Macrophomi- } \\
\text { napha seolina }\end{array}$ & $\begin{array}{l}\text { Fusarium } \\
\text { oxysporum }\end{array}$ & $\begin{array}{l}\text { Aspergillus } \\
\text { flavus }\end{array}$ & $\begin{array}{l}\text { Phoma } \\
\text { exigua }\end{array}$ & $\begin{array}{l}\text { Colletotri- } \\
\text { chum spp }\end{array}$ & $\begin{array}{l}\text { Rhizopus } \\
\text { stolonifer }\end{array}$ & $\begin{array}{l}\text { Penicillium } \\
\text { spp }\end{array}$ & $\begin{array}{c}\text { Cercospora } \\
\text { spp }\end{array}$ & $\begin{array}{l}\text { Alternaria } \\
\text { Alternata }\end{array}$ \\
\hline $\mathrm{T}_{0}$ (Control) & $40 \mathrm{a}$ & $46 a$ & $\begin{array}{c}12 \mathrm{a} \\
(20.27)\end{array}$ & $56 a$ & $54 \mathrm{a}$ & $\begin{array}{c}6 \mathrm{a} \\
(14.18)\end{array}$ & $\begin{array}{c}6 \mathrm{a} \\
(14.18)\end{array}$ & $\begin{array}{c}4 a \\
(11.54)\end{array}$ & $\begin{array}{c}8 \mathrm{a} \\
(16.43)\end{array}$ & $\begin{array}{c}4 a \\
(11.54)\end{array}$ & $\begin{array}{c}2 \mathrm{a} \\
(8.13)\end{array}$ \\
\hline $\begin{array}{l}\mathrm{T}_{1}(200 \mathrm{ppm} \\
\mathrm{CS})\end{array}$ & $\begin{array}{c}0 \mathrm{c} \\
(0.7)\end{array}$ & $40 \mathrm{~b}$ & $\begin{array}{c}0 \mathrm{~b} \\
(0.7)\end{array}$ & $\begin{array}{c}20 \mathrm{~b} \\
(26.56)\end{array}$ & $40 \mathrm{~b}$ & $\begin{array}{c}0 \mathrm{~b} \\
(0.7)\end{array}$ & $\begin{array}{c}0 \mathrm{~b} \\
(0.7)\end{array}$ & $\begin{array}{c}4 a \\
(11.54)\end{array}$ & $\begin{array}{c}2 \mathrm{~b} \\
(8.13)\end{array}$ & $\begin{array}{c}0 \mathrm{~b} \\
(0.7)\end{array}$ & $\begin{array}{c}0 \mathrm{~b} \\
(0.7)\end{array}$ \\
\hline $\mathrm{T}_{2}(500 \mathrm{ppm}$ & $0 \mathrm{c}$ & $10 f$ & $0 \mathrm{~b}$ & $0 \mathrm{c}$ & $35 c$ & $0 \mathrm{~b}$ & $0 \mathrm{~b}$ & $2 \mathrm{c}$ & $1 \mathrm{c}$ & $0 \mathrm{~b}$ & $0 \mathrm{~b}$ \\
\hline CS) & $(0.7)$ & $(18.43)$ & $(0.7)$ & $(0.7)$ & $3 x$ & $(0.7)$ & $(0.7)$ & $(8.13)$ & $(5.74)$ & $(0.7)$ & $(0.7)$ \\
\hline $\mathrm{T}_{3}(1000 \mathrm{ppm}$ & $0 \mathrm{c}$ & $5 \mathrm{~g}$ & $0 \mathrm{~b}$ & $0 \mathrm{c}$ & $15 \mathrm{e}$ & $0 \mathrm{~b}$ & $0 \mathrm{~b}$ & $1 d$ & $1 \mathrm{c}$ & $0 \mathrm{~b}$ & $0 \mathrm{~b}$ \\
\hline CS) & $(0.7)$ & (12.92) & $(0.7)$ & $(0.7)$ & (22.79) & $(0.7)$ & $(0.7)$ & $(5.74)$ & $(5.74)$ & $(0.7)$ & $(0.7)$ \\
\hline $\mathrm{T}_{4}(2000 \mathrm{ppm}$ & $0 \mathrm{c}$ & $\mathrm{Oh}$ & $0 \mathrm{~b}$ & $0 \mathrm{c}$ & $5 f$ & $0 \mathrm{~b}$ & $0 \mathrm{~b}$ & $4 a$ & $0 \mathrm{~d}$ & $0 \mathrm{~b}$ & $0 \mathrm{~b}$ \\
\hline CS) & $(0.7)$ & $(0.7)$ & $(0.7)$ & $(0.7)$ & (12.92) & $(0.7)$ & $(0.7)$ & (11.54) & $(0.7)$ & $(0.7)$ & $(0.7)$ \\
\hline $\mathrm{T}_{5}$ (200ppm & $20 b$ & $40 \mathrm{~b}$ & $0 \mathrm{~b}$ & $0 \mathrm{c}$ & $25 \mathrm{~d}$ & $0 \mathrm{~b}$ & $0 \mathrm{~b}$ & $3 b$ & $0 \mathrm{~d}$ & Ob & $0 \mathrm{~b}$ \\
\hline YES) & $(26.56)$ & 400 & $(0.7)$ & $(0.7)$ & $25 d$ & $(0.7)$ & $(0.7)$ & $(9.97)$ & $(0.7)$ & $(0.7)$ & $(0.7)$ \\
\hline $\mathrm{T}_{6}(500 \mathrm{ppm}$ & $0 \mathrm{c}$ & $30 \mathrm{c}$ & $0 \mathrm{~b}$ & $0 \mathrm{c}$ & 20d & $0 \mathrm{~b}$ & $0 \mathrm{~b}$ & $3 b$ & $0 \mathrm{~d}$ & $0 \mathrm{~b}$ & $0 \mathrm{~b}$ \\
\hline YES) & $(0.7)$ & $30 \mathrm{c}$ & $(0.7)$ & $(0.7)$ & $(26.56)$ & $(0.7)$ & $(0.7)$ & (9.97) & $(0.7)$ & $(0.7)$ & $(0.7)$ \\
\hline $\mathrm{T}_{7}(1000 \mathrm{ppm}$ & $0 \mathrm{c}$ & $25 \mathrm{~d}$ & $0 \mathrm{~b}$ & $0 \mathrm{c}$ & $15 \mathrm{e}$ & $0 \mathrm{~b}$ & $0 \mathrm{~b}$ & $2 \mathrm{c}$ & $0 \mathrm{~d}$ & $0 \mathrm{~b}$ & $0 \mathrm{~b}$ \\
\hline YES) & $(0.7)$ & $25 \mathrm{~d}$ & $(0.7)$ & $(0.7)$ & (22.79) & $(0.7)$ & $(0.7)$ & $(8.13)$ & $(0.7)$ & $(0.7)$ & $(0.7)$ \\
\hline $\mathrm{T}_{8}(2000 \mathrm{ppm}$ & $0 \mathrm{c}$ & $15 \mathrm{e}$ & $0 \mathrm{~b}$ & $0 \mathrm{c}$ & $0 \mathrm{~g}$ & $0 \mathrm{~b}$ & $0 \mathrm{~b}$ & $0 \mathrm{e}$ & $0 \mathrm{~d}$ & $0 \mathrm{~b}$ & $0 \mathrm{~b}$ \\
\hline YES) & $(0.7)$ & $(22.79)$ & $(0.7)$ & $(0.7)$ & $(0.7)$ & $(0.7)$ & $(0.7)$ & $(0.7)$ & $(0.7)$ & $(0.7)$ & $(0.7)$ \\
\hline $\mathrm{T}_{9}$ (Vitavax-200 & $0 \mathrm{c}$ & $\mathrm{Oh}$ & $0 \mathrm{~b}$ & $0 \mathrm{c}$ & $0 \mathrm{~g}$ & $0 \mathrm{~b}$ & $0 \mathrm{~b}$ & $0 \mathrm{e}$ & $0 \mathrm{~d}$ & $0 \mathrm{~b}$ & $0 \mathrm{~b}$ \\
\hline WP) & $(0.7)$ & $(0.7)$ & $(0.7)$ & $(0.7)$ & $(0.7)$ & $(0.7)$ & $(0.7)$ & $(0.7)$ & $(0.7)$ & $(0.7)$ & $(0.7)$ \\
\hline $\mathrm{LSD}_{0.05}$ & 1.12 & 1.87 & 0.178 & 1.52 & 1.61 & 0.357 & 0.107 & 0.544 & 0.240 & 0.274 & 0.178 \\
\hline $\mathrm{CV}(\%)$ & 9.10 & 4.67 & 4.00 & 10.15 & 3.95 & 10.15 & 3.18 & 4.09 & 3.53 & 9.04 & 7.23 \\
\hline
\end{tabular}

Values within the same column having a common letter(s) do not differ significantly ( $\mathrm{P} \geq 0.01)$

CS indicates Chitosan Solution, YES indicates Yeast Elicitor Solution

Figures in the parentheses are arcsine transformed values

\section{Discussion}

The present investigation has been carried out by seed priming of vegetable seeds with different elicitors such as Chitosan and Yeast Elicitor. Altogether fourteen fungi, representing twelve genera were recorded in the seeds of bottle gourd, sweet gourd, snake gourd, wax gourd and cucumber collected from seed traders of Mymensingh districts.

In Bottle gourd seeds, eight fungi were detected. Seed priming by Chitosan @ 2000 ppm and Yeast elicitor @ $1000 \mathrm{ppm} \& 2000 \mathrm{ppm}$ and seed treatment with $0.35 \%$ Vitavax-200 WP significantly reduced seed-borne fungal pathogens followed by Chitosan @ 1000 ppm \& 500 $\mathrm{ppm}$. In Sweet gourd seeds, ten fungi were detected. Seed priming with Chitosan @ 2000 ppm, Yeast Elicitor (a) 2000 ppm and Vitavax-200 WP significantly reduced seed-borne fungal pathogens followed by Yeast Elicitor (a) 500 ppm, 1000 ppm \& 2000 ppm. The present findings was supported by Begum and Momin (2000); Kamble et al. (1999) where they also reported the association of similar fungi viz. Aspergillus flavus, Penicilium spp., Fusarium spp., Rhizopus spp. with vegetable seeds. In Snake gourd seed, eight fungi were detected. Seed priming by Chitosan @ 2000 ppm significantly reduced seed-borne fungal pathogens followed by Yeast Elicitor Solution @ 200 ppm, 500 ppm, 1000 ppm \& 2000 ppm. In Wax gourd seed, ten fungi were detected. Seed priming by Chitosan @ 2000 ppm significantly reduced seed-borne fungal pathogens followed by Chitosan@ 1000 ppm \& Yeast Elicitor @ $2000 \mathrm{ppm}$. The present findings was supported by Begum and Momin (2000) where they also reported the association of similar fungi such as Aspergillus flavus, Penicilium spp., Fusarium spp., Rhizopus spp. with vegetable seeds. In Cucumber, eleven fungi were detected. Seed priming by Yeast Elicitor @ 2000 ppm significantly reduced seed-borne fungal pathogens followed by Chitosan @ 2000 ppm. The present findings was supported by Nasreen and Sultana (2000); Kamble et al. (1999) and Braccini and Dhingara (1996) where they also reported the association of similar fungi viz. Rhizoctonia spp., Colletotrichum sp., Fusarium spp., Alternaria spp., Macrophomina phaseolina, Aspergillus niger, Penicillium spp. and Rhizopus spp. with vegetable seeds.

Present investigation indicates that seed priming of cucurbits by elicitor can suppress the growth of seedborne fungi as the elicitor may induce resistance against pathogens. All doses of Chitosan solution shown reduction in seed-borne fungal infection in bottle gourd, sweet gourd, snake gourd, wax gourd and cucumber. Chitosan $(2000 \mathrm{ppm})$ significantly reduced the seedborne fungal pathogens. The findings of the present investigation are in agreement with Zheng et al. (2012), who reported that, chitosan coating increased seed germination, plant growth and soybean yield efficiently. Tingda et al. (1994) reported that, $0.1 \%$ chitosan help for the growth stimulation of cotton and maize seeds. Alam et al. (2014) also reported that, 1\% chiosan solution stimulate the germination percentage of chili seed and control seed-borne fungi associated with chilli seed. Similarly, seed priming with Yeast Elicitor solution showed that all doses of Yeast Elicitor solution reduced seed-borne fungal infection of cucurbits Yeast 
Elicitor (2000 ppm) significantly reduced the seed-borne fungal pathogens which is statistically similar to the 0.35\% Vitavax-200 WP. Al- Tawaha et al. (2011) reported that foliar application of yeast extract hold promises for increasing the seed yield and isoflavone content of soybean seeds. Moreover, due to natural sources both elicitors are environmentally safe.

\section{Conclusion}

Chitosan and Yeast Elicitor are potential compounds for priming of seed to control seed borne fungi of Cucurbits. Chitosan and yeast elicitor are bio-polymers and not harmful for ecosystem and completely safe for human health. Therefore, application of chitosan and yeast elicitor can be utilized as seed- treating bio-polymer in replace to chemical pesticides. The effectiveness of these products should also be assessed against seedborne fungi of cucurbits for their commercial application.

\section{Acknowledgement}

The study was jointly funded by the Ministry of Science and Technology, The peoples' Republic of Bangladesh for awarding NST fellowship and Bangladesh Agricultural University Research System (BAURES), Bangladesh Agricultural University, Mymensingh, Bangladesh.

\section{References}

Alam, M. Z., Hamim, I., Ali, M. A. and Ashrafuzzaman M. 2014 Effect of seed treatment on seedling health of chilli. J. Environ. Sci. \& Natural Resources 7(1):177-181.

Al-Tawaha, A.R.M., 2011. Effects of soil type and exogenous application of yeast extract on soybean seed isoflavone concentration. Int. J. Agric. Biol., 13: 275-278.

Ari, S. and Cakir, O. 2009. Defensive and secondary metabolism in Astragalus chrysochlorus cell cultures in response to yeast extract stressor. J. Environ. Biol 30(1): 51-55.

Begum, H. A. and Momin, A. 2000. Comparison between two detection techniques of seed borne pathogens in cucurbits in Bangladesh. Pakistan Journal of Scientific and Industrial Res 43(4): 244-248.

Boonlertnirun, S., Boonreung, C., Suvansara, R. 2008. Application of Chitosan in Rice Production. Journal of Metals, Materials and Minerals 18: 47-52.

Boller, T., 1995. Chemoperception of microbial signals in plant cells. Annu. Rev. Plant Physiol.Plant Mol. Biol. 46, 189-214.

Braccini, A. L. and Dhingara, O. D. 1996. Identification of fungi associated with soybean and cucumber seeds by different detection method. Department of Agronomy, University of Estadual de Maringa.18 (3): 495-503.

Cai, Z., Kastell, A., Mewis, I., Knorr, D., Smetanska, I. 2012. Polysaccharide elici-tors enhance anthocyanin and phenolic acid accumulation in cell suspension cultures of Vitis vinifera.Plant Cell Tissue Org. Cult. 108, 401-409.

Ellis, M. B. 1971. Dematiaceous hyphomycetes. Commonwealth Mycological Institute, England. 507 pp.

Fakir, G.A. 1983. Teaching, research and training activities on seed pathology in Bangladesh. Seed Sci Technol 11:1345-1352.

Islam, S. M. A., Hossain, I., Fakir, G. A. and Asad-ud-doullah, M. 2001. Effect of physical seed sorting, seed treatment with garlic extract and vitavax 200 on seed borne fungal flora and seed yield of jute (Corchorus capsularis L.). Pakistan Journal of Biological Science. 4 (12), 1509-1511.

Mondal, M. M. A., Ismail, M. R., Malek, M. A. and Puteh, A. B. 2013. Foliar Application of Chitosan on Growth and Yield Attributes of Mungbean (Vigna radiata). Bangladesh J.Bot 42 (1): 179-183.

ISTA (International Seed Testing Association). 2006. International Rules for Seed Testing. Rules amendments. Seed Science and Technology 29 (2): 1-127.

Kamble, P., Borkar, G. M. and Patil, D. V. 1999. Studies on seedborne pathogens of pumpkin, cucumber, water melon and musk melon. J. Soils and Crops 9 (2):234-238.

Khokon, M. A. R., Hossain, M. A., Muenmasa, S., Uraji, M., Nakamura, Y., Mori, I. C. and Murata, Y. 2010. Yeast elicitor-induced stomatalclosure along with peroxidasemediated ROS production in arabidopsis. Plant and Cell Physiology 57(11):1915-1921.

Nasreen, S. and Sultana, N. 2000. Seed health testing of cucumber (Cucumis sativus L.). Pakistan Journal of Scientific and Industrial Research 43(2): 127-129.

Neergaard, P. 1979. Seed Pathology. Vol 1. The Macmillan Press Ltd., 839 p.

Prakash, G., Srivastava, A.K. 2008. Statistical elicitor optimization studies for the enhancement of azadirachtin production in bioreactor Azadirachtaindica cell cultivation. Biochem. Eng. J. 40, 218-226.

Putalun, W., Luealon, W., De-Eknamkul, W., Tanaka, H., Shoyama, Y. 2007. Improvement of artemisinin production by chitosan in hairy root cultures of Artemisiaannua. Biotechnol. Lett. 29, 1143-1146.

Tingda, J., Ruixia, M., Ruiming, Q., Chunping, Z. 1994. Seed treatment and incubation of plant pathology and chitosan. Environmental Science 6: 112-115.

Yarahmadi, M., Azizi, M., Safaei, Z. 2014. Study on the effect of Chitosan, vanillin and acetic acid on fungal diseases control of Rhizopus stolonifer in strawberry fruits in-vitro and invivo. European Journal of Experimental Biology 4(3): 219225.

Zeng, D., Luo, X., and Tu, R. 2012. Aaication of bioactive chitin based on chitosan for soybean seed protection. International Journal of Carbohydrate Chemistry. 1-5.

Zhao, J., Zhou, L., Wu, J., 2010. Effects of biotic and abiotic elicitors on cell growth and tanshinone accumulation in Salvia miltiorrhiza cell cultures. Appl. Microbiol.Biotechnol. 87, $137-144$. 